\title{
Research Article \\ A histopathological review of nephrectomy specimens with an emphasis on pathological assessment of prognostic indicators in malignant lesions
}

\author{
Shubha P. Bhat ${ }^{1}$, Krishna Prasad H.V. ${ }^{2}$, Rajeev T.P. ${ }^{3}$, Kishan Prasad H.L. ${ }^{4}$, Teerthanath Srinivas ${ }^{5}$, Jayaprakash Shetty K. ${ }^{6}$ \\ ${ }^{1}$ Associate Professor, ${ }^{2}$ Assistant Professor, ${ }^{4}$ Additional Professor, ${ }^{5,}$ Professor, Department of Pathology; ${ }^{3}$ Professor, Department of \\ Urology, K.S Hegde Medical Academy Nitte (Deemed to be University), Mangalore, Karnataka, 575018, India
}

(Received: October $2020 \quad$ Revised: November $2020 \quad$ Accepted: November 2020)

Corresponding author: Shubha P. Bhat. Email: bhatshubha_257@rediffmail.com

\begin{abstract}
Introduction and Aim: Various non-neoplastic and neoplastic lesions occur in the kidney. Pathological assessment of gross and microscopic features in nephrectomy specimens is essential for diagnosis and predicts the prognosis in malignant tumours.

Materials and Methods: Case records of 46 nephrectomy specimens received between two-year periods were retrieved. Detailed gross and histopathological and immunohistochemical features were studied, and malignant tumours were analysed using CAP protocol.

Results: Out of 46 nephrectomy specimens, 17 were non-neoplastic and 29 neoplastic. Males constituted 32 cases and females 14 cases. The commonest non-neoplastic kidney lesion was chronic pyelonephritis with hydronephrosis (29\%). The mixed epithelial and stromal tumour was the frequently encountered benign tumour (50\%). Renal cell carcinoma was the most common malignant tumour in adults (70\%) and Wilms tumour in children (4\%).

Conclusion: Histopathological examination of nephrectomy specimens helps in diagnosing, staging, and planning the management.
\end{abstract}

Keywords: Nephrectomy; prognosis; pyelonephritis; neoplasm

\section{INTRODUCTION}

$\mathrm{K}$ idneys are involved by various non-neoplastic and neoplastic lesions requiring removal (1). mon non-neoplastic lesions are chronic pyelonephritis (CPN) and hydronephrosis (HDN), while renal cell carcinoma (RCC) and Wilms' tumour are the commonest renal malignancies in adults and children, respectively. Both benign and malignant tumours occur in the kidney, of which the latter is more common (2). The incidence of renal cancers is high in Western and developed countries and low in Eastern and developing countries. However, the exact cause for this difference is not clearly understood (3). Renal cell carcinoma represents 2 to $3 \%$ of all adult visceral malignancies and accounts for $90 \%$ of adults' renal cancers. They occur most commonly in the sixth to seventh decade of life, with a male preponderance (2). Common clinical presentations include flank pain, palpable mass, and painless haematuria (4). Widespread use of non-invasive imaging modalities like ultrasonography (USG), Computed tomography (CT)scan, and magnetic resonance imaging (MRI) have led to increased detection of incidental renal mass (5).Random urine cytology performed on three nonconsecutive days increases the sensitivity of the detection of upper tract urothelial carcinomas (UTUC) (6). Core needle biopsy (CNB) or fine-needle aspiration cytology of the renal mass helps determine RCC's subtypes (7). Simple nephrectomy is the treatment of choice for irreversibly damaged, nonfunctioning kidneys (8). Radical nephrectomy is the treatment of choice for malignant tumours (9). Nephron sparing surgeries or partial nephrectomy is indicated in bilateral RCC or RCC involving a solitary functioning kidney and small RCC less than $4 \mathrm{~cm}$ in size $(5$, 9).Laparoscopic and robotic approaches have significantly reduced the morbidity of partial nephrectomy procedures compared to open approaches (10). Histopathological examination of nephroureterectomy specimens is of utmost importance for confirmation of the diagnosis. It also helps assess the prognostic factors like tumour size, histologic subtype, percentage of tumour necrosis, tumour grade, and stage in malignant tumours, which are the determinants of the patient's survival rate (11).

In this retrospective study, we evaluated the histopathological spectrum of lesions in nephrectomy specimens. We assessed the histologic type, grading, and pathological stage of neoplastic lesions using the World Health Organization (WHO) 2016 classification.

\section{MATERIALS AND METHODS}

This is a retrospective study done in a tertiary care hospital in Mangalore, India. Ethical clearance for the 
study was obtained from the institutional ethics committee. The case records of 46 nephrectomy specimens received during the two years between June 2018 and May 2020 were retrieved. Details about the demographic profile, presenting complaints, imaging findings, and the type of surgery were noted and analysed. Detailed gross and histopathological features were studied, and lesions were broadly categorized as non-neoplastic and neoplastic. Non-neoplastic lesions were assessed grossly for size, scars, dilation of pelvicalyceal system, thinning of cortex, cysts, calculi, and presence of pus/necrotic material. Urine cytology by the Paris system (12) and CNB findings of renal mass was also noted when performed. In neoplastic lesions, the location and size of the tumour in the kidney were noted. Specimens were examined for ureteric margin, renal sinus invasion (fat, loose connective tissue or endothelial lined spaces), hilar soft tissue, perinephric fat, renal vein, adrenal and Gerota's fascia invasion, grossly and microscopically. Renal parenchymal and renal capsular margins were assessed in partial nephrectomy specimens. The histological typing of neoplastic lesions was done according to theWHO2016 classification of tumours of the urinary system. The WHO/International Society of Urological Pathology (ISUP) grading system was used to grade clear cell RCC and papillary RCC. Malignant tumours were analysed using the College of American Pathologist (CAP)protocol June 2017 template, and the pathological stage of RCC was done by using Tumour, Node, and Metastasis (TNM) classification of American Joint Committee on Cancer (AJCC) $8^{\text {th }}$ edition. The children's' oncology group staging was used to stage Wilms' tumour. Immunohistochemistry (IHC) was performed wherever necessary. The results were represented as frequency and percentage.

\section{RESULTS}

The study involved 46 nephrectomy specimens in our tertiary care hospital, including 16 cases of simple open nephrectomy and one partial nephrectomy performed for non-neoplastic conditions, and 25 cases of radical nephrectomy and four partial nephrectomies performed for neoplastic conditions. The youngest patient in our study was an eight-month-old, and the oldest was 80years-old. Males constituted 32 cases (70\%) and females 14 cases (30\%). Non neoplastic lesions comprised $37 \%$ (17cases) and neoplastic 63\% (29cases). Most of the non-neoplastic lesions were seen in the $7^{\text {th }}$ decade of life (5cases) followed by the $6^{\text {th }}$ and $3^{\text {rd }}$ decade (3cases each). Neoplastic lesions occurred commonly in the $7^{\text {th }}$ decade $(31 \%)$, followed by the $5^{\text {th }}$ and $6^{\text {th }}$ decade ( $28 \%$ and $21 \%$, respectively).

Overall, flank pain was the most common complaint noted in both non-neoplastic and neoplastic lesions, which accounted for $60.8 \%$ of cases, followed by haematuria $(32.6 \%)$ and nocturia (15.2\%). Other complaints were mass per abdomen, fever, burning micturition, poor urine stream, dysuria, loss of appetite, and weight. A history of penetrating trauma was noted in one case. Grossly, in non-neoplastic lesions, the kidney's size was normal in 12\%, enlarged in 59\% cases, and contracted in 29\%. Dilation of the pelvicalyceal system was seen in $76 \%$ of cases, thinning the cortex in $47 \%$, renal stones in $29 \%$, pus/necrotic material in $18 \%$, and cysts in $12 \%$ of cases. Table 1 shows the distribution of non-neoplastic lesions in our study.

Table 1: Non-neoplastic lesions

\begin{tabular}{|c|c|}
\hline Lesions & $\begin{array}{c}\text { Number (n=17) } \\
(\mathbf{\%})\end{array}$ \\
\hline CPN & $3(18)$ \\
\hline CPN with hydronephrosis & $9(52)$ \\
\hline CPN with pyonephrosis & $2(12)$ \\
\hline $\begin{array}{c}\text { Xanthogranulomatous pyelonephritis } \\
\text { with hydronephrosis }\end{array}$ & $1(6)$ \\
\hline Acute pyelonephritis & $1(6)$ \\
\hline Multicystic renal dysplasia & $1(6)$ \\
\hline
\end{tabular}

Urine cytology was performed and assessed in seven cases of the neoplastic lesion. There was a correlation with histopathological diagnosis in four patients. Two high-grade urothelial carcinomas (HGUC) cases of the renal pelvis were reported as negative for HGUC. Pelviureteric cavernous haemangioma was reported as suspicious of HGUC, which showed reactive atypia of renal pelvic epithelium on resection.

Ultrasound-guided CNB was done in two cases, one case each of oncocytoma and papillary RCC. Cystoscopy biopsy was done in the HGUC of the distal ureter. Out of the 29 neoplastic lesions, six patients (20.6\%) were benign, and 23 cases (79.3\%) malignant. Among benign tumours, we had 3 cases of mixed epithelial and stromal tumour (MEST), and one case each of angiomyolipoma, oncocytoma, and cavernous haemangioma of the pelviureteric region.

Table 2: Types of RCC

\begin{tabular}{|c|c|}
\hline Lesions & Number (n=17) (\%) \\
\hline Clear cell & $12(75 \%)$ \\
\hline Papillary & $2(13 \%)$ \\
\hline Chromophobe & $1(6 \%)$ \\
\hline $\begin{array}{c}\text { Multilocular cystic renal neoplasm } \\
\text { of low malignant potential }\end{array}$ & $1(6 \%)$ \\
\hline
\end{tabular}

Out of the 23 malignant tumours, RCC was the most common (16cases, 70\%), followed by infiltrating urothelial carcinoma of renal pelvis and ureter (6cases, $26 \%$ ), and Wilms' tumour (1case,4\%). Table 2 shows various types of RCC in our study. In the WHO/ISUP grading of clear cell and papillary RCC, grade 1 
tumours were most frequent (47\%) followed by grade 2 $(33 \%)$ and grade $3(20 \%)$. The majority of the RCCs $(62 \%)$ in our study did not show tumour necrosis. The majority of RCC were pT1(56\%) followed by pT2 $(25 \%)$ and pT3(19\%). We had 3 cases of HGUC of the renal pelvis, two were pT3, and one patient was pT4 pN2 with lympho-vascular invasion. One case each of proximal (high grade), mid (low grade), and distal ureter (high grade) urothelial carcinoma was noted. Wilms' tumour was in stage 3. Regarding surgical resected margins of malignant tumours, 4 cases showed positive margins. These were; chromophobe RCC showing renal sinus vessel invasion, HGUC of the renal pelvis showing renal vein invasion at the hilar margin, Wilms' tumour showing invasion of Gerota's fascia and renal sinus soft tissue, low-grade urothelial carcinoma of mid ureter showing adventitial tumour deposits at the ureteric margin. One case each of the chromophobe RCC and HGUC pelvis showed metastatic tumour deposits in the lymph node.

\section{DISCUSSION}

In India, the estimated incidence of RCC among males is about $2 / 100,000$ population and among females is about $1 / 100,000$ population (3). Neoplastic lesions predominated over non-neoplastic lesions in our study. Most non-neoplastic lesions are related to calculi, which can be managed conservatively, hence avoiding surgery. Renal neoplasm is a disease of elderly males usually associated with risk factors like hypertension, type 2 diabetes mellitus, obesity, and smoking (17). In our study, neoplastic lesions were common in the $7^{\text {th }}$ decade of life (14 cases) with male preponderance.

Simple nephrectomy involves removing the kidney without Gerota's fascia, adrenals, or lymph node dissection (8). In the present study, out of 17 nonneoplastic lesions, nine patients underwent simple open nephrectomy and seven patients by laparoscopic approach. One patient who underwent partial nephrectomy was a two-year-old boy with left kidney duplex with upper moiety pyonephrosis.

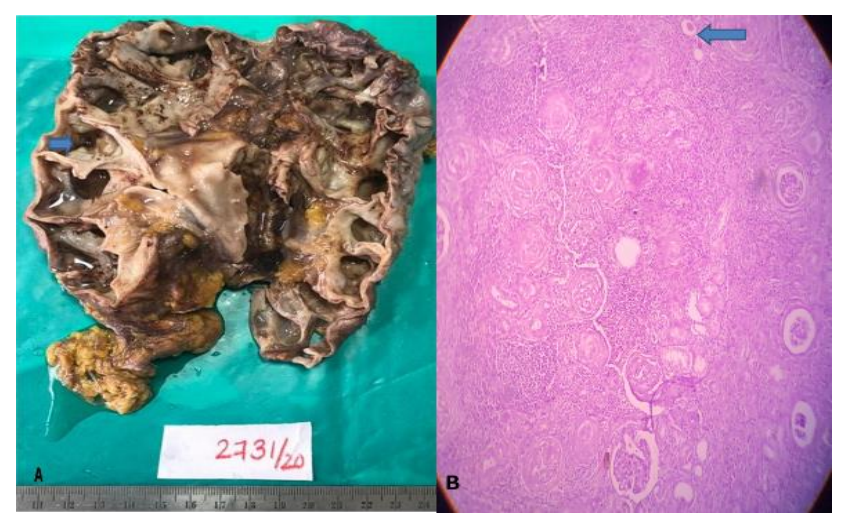

Fig. 1: Chronic pyelonephritis with hydronephrosis A- Gross specimen showing dilation of the pelvicalyceal system (arrow) with thinning of the cortex. B- Microscopy showing tubular atrophy thyroidisation (arrow), periglomerular sclerosis, fibrosis and chronic inflammation, $\mathrm{H} \& \mathrm{E}, 10 \mathrm{x}$

Pyelonephritis refers to inflammation involving renal tubules, interstitium, and renal pelvis. Grossly, CPN due to vesicoureteral reflux shows polar scars and those due to obstruction, showing diffuse involvement of pyramids (13). In the present study, CPN with HDN was the most frequent non-neoplastic lesion (52\%). All of them had calculus and showed diffuse and coarse corticomedullary scars, along with dilated and deformed pelvicalyces (Fig. 1a). Rest five cases of CPN without evidence of obstruction showed polar scars with blunted calyces, which may be attributed to vesicoureteral reflux. Microscopically, there will be interstitial infiltrates of lymphocytes, plasma cells, tubular dilation, and atrophy with hyaline casts (thyroidisation), periglomerular sclerosis, and interstitial fibrosis (Fig. 1b; 14).

There were 10 cases of HDN in the present study. Nine cases occurred with CPN and one case with XPN. There was a male preponderance with most of the cases occurring in the $7^{\text {th }}$ decade. The youngest patient was a two-year-old boy with left kidney duplex with upper moiety pyonephrosis and hydroureteronephrosis.

Collecting a random voided urine sample for three nonconsecutive days will increase the sensitivity of preoperative grading in upper tract urothelial carcinoma (UTUC), prompting conservative management in low grade, low stage tumours (6). Our study's discrepancies in urine cytology diagnosis may be attributed to reporting on one and two-day urine samples and heterogeneity of grade within the tumour.

Core needle biopsy is useful for diagnosing the renal lesion and stratifying the risk of active surveillance in patients found to have RCC (15). Most discrepancies in diagnosis are attributed to small sample size, tumour heterogeneity, and certain diagnostic groups, particularly oncocytic neoplasms (7). In the present study, CNB was performed in one case each of oncocytoma and papillary RCC which were diagnosed as an eosinophilic variant of RCC and clear cell RCC, respectively. The patient with oncocytoma had a posterior iliac crest and sacral lesion, suspected to be metastasis on a computerized tomography scan; hence we reported eosinophilic variant clear cell RCC on CNB and advised IHC. Table 3 shows IHC findings in renal oncocytoma in our study.

In the present study, out of 29 neoplastic lesions, radical nephrectomy was done in 25cases. Partial nephrectomy was performed in 4 cases; all were pT1cortical tumours with small tumour size and exophytic. One patient had a solitary kidney. 
Table 3: Immunohistochemistry findings in neoplastic lesions

\begin{tabular}{|c|c|c|c|}
\hline $\begin{array}{c}\text { IHC } \\
\text { markers }\end{array}$ & $\begin{array}{c}\text { Renal } \\
\text { oncocytoma }\end{array}$ & $\begin{array}{c}\text { Chromophobe RCC with } \\
\text { sarcomatoid transformation }\end{array}$ & $\begin{array}{c}\text { Wilms' } \\
\text { tumour }\end{array}$ \\
\hline Pan CK & - & Positive & Positive \\
\hline CK 7 & Negative & Positive & - \\
\hline CD10 & Negative & Negative & - \\
\hline RCC ma & Negative & Negative & - \\
\hline CD117 & Positive & Positive & - \\
\hline Vimentin & Negative & Positive in spindle cells & - \\
\hline ki 67 & - & - & $30 \%$ \\
\hline WT 1 & - & - & Positive \\
\hline
\end{tabular}

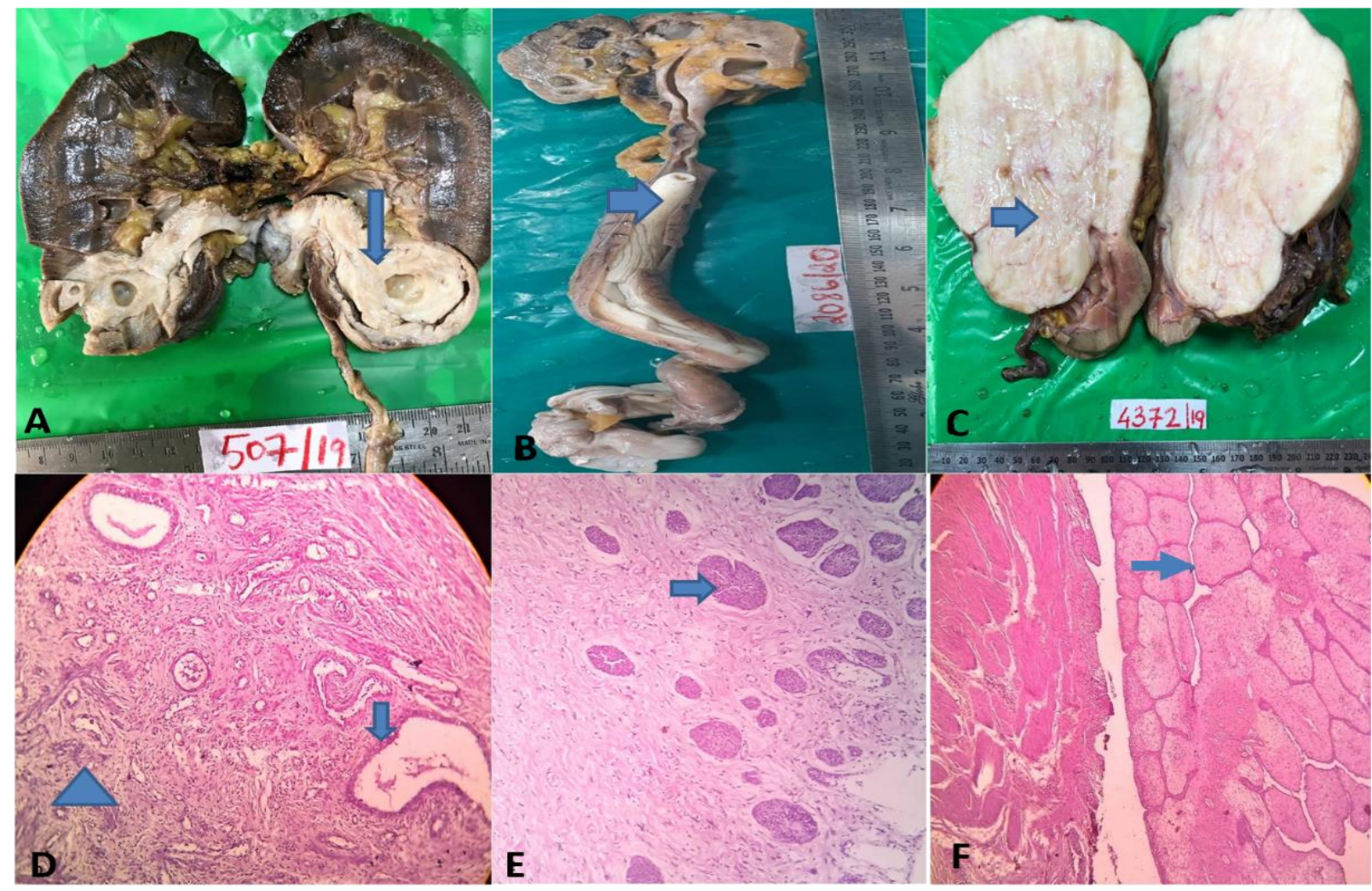

Fig. 2: MEST A- Gross specimen showing a well-circumscribed tumour involving the cortex and medulla (arrow). B-completely detached from the pelvis into the ureter(arrow).C- exophytic mass from the cortex(arrow). D-Glands lined by low columnar cells (arrow), surrounded by ovarian type stroma (arrowhead) H\&E, 10x. E- urothelial lining of glands (arrow) H\&E, 10x. F- phyllodes like architecture (arrow), H\&E, 10x.

Mixed epithelial and stromal tumours are related to hormonal imbalance and typically occur in perimenopausal women (8). Out of the three MEST cases, two were perimenopausal women, and one was a 55-year-old male. Fig. 2A, $2 \mathrm{~B}$ and $2 \mathrm{C}$ show gross findings, and Fig. 2D, 2E, and 2F show MEST's microscopic features. Renal oncocytoma is a benign epithelial tumour occurring commonly in the $7^{\text {th }}$ decade with male preponderance (8). In the present study, we had a case of oncocytoma in a 48year old male. Fig. 3A and $3 \mathrm{~B}$ show gross and microscopic features of renal oncocytoma, respectively. Oncocytoma is typically positive for CD 117 (Fig. 3C), Vimentin (73\%cases), and $\mathrm{PR}(90 \%)$ and negative for CD 10, CK7, and RCC marker. Differential diagnoses include SDH deficient RCC and the eosinophilic variant of chromophobe RCC. While the former is negative for SDHB, CK 7, and CD117, the latter is positive for these markers and RCC marker and negative for vimentin and CD 10. A small subset of tumours has overlapping histology between oncocytoma and Chromophobe RCC called hybrid oncocytic/chromophobe tumour $(8,16)$. Cavernous haemangioma is an uncommon benign vascular tumour of the kidney occurring in adults. They commonly occur in the renal hilum (8). Fig. 4A shows the gross findings of cavernous haemangioma of the pelviureteric region. Fig. 4B shows the microscopic findings of the same. 


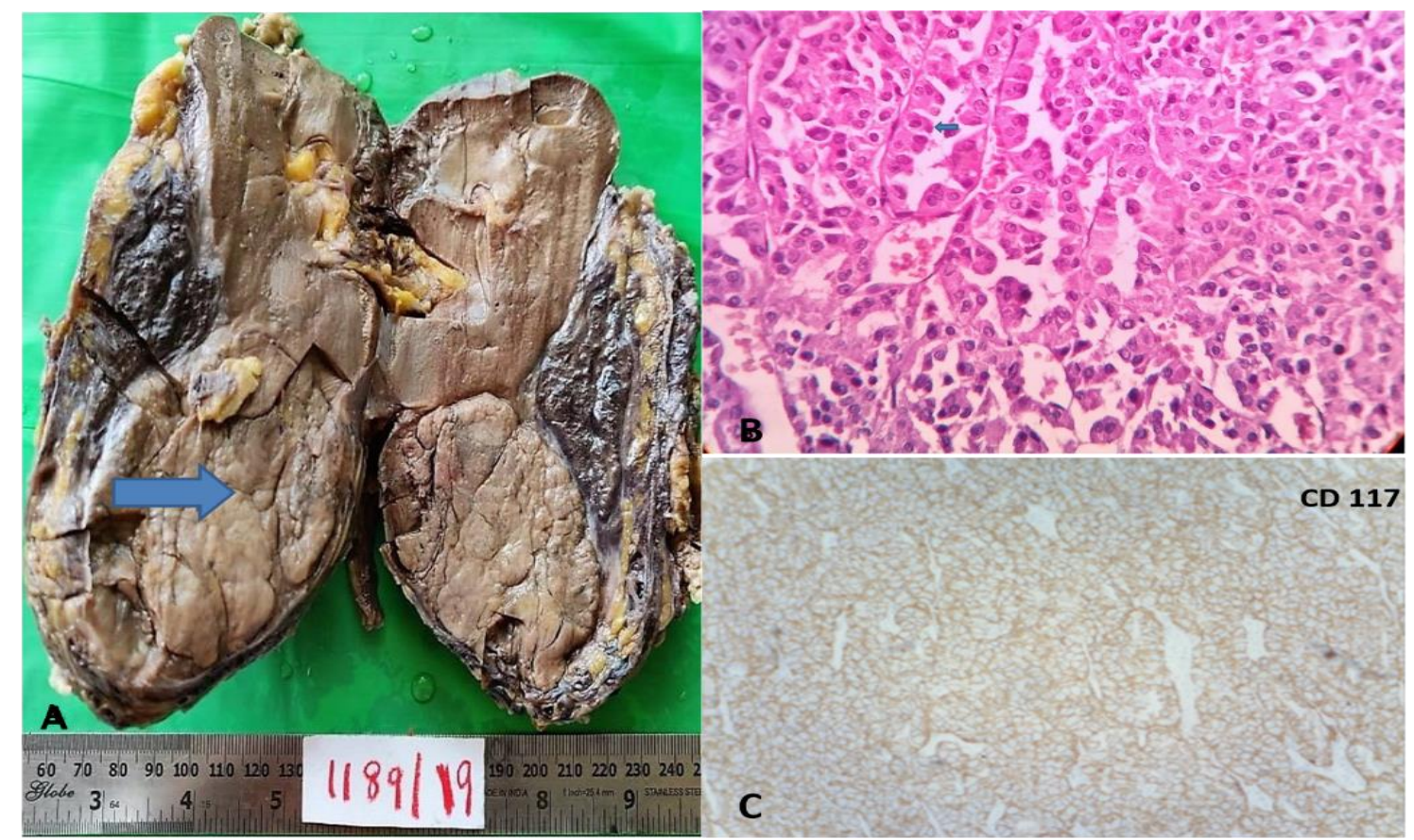

Fig. 3: Renal oncocytoma A-Gross specimen showing mahogany brown, solid, and well-circumscribed tumour (arrow). B- oncocytic cells containing dense granular cytoplasm with regular round nuclei (arrow).C-CD 117 showing complete membrane staining of tumour cells.

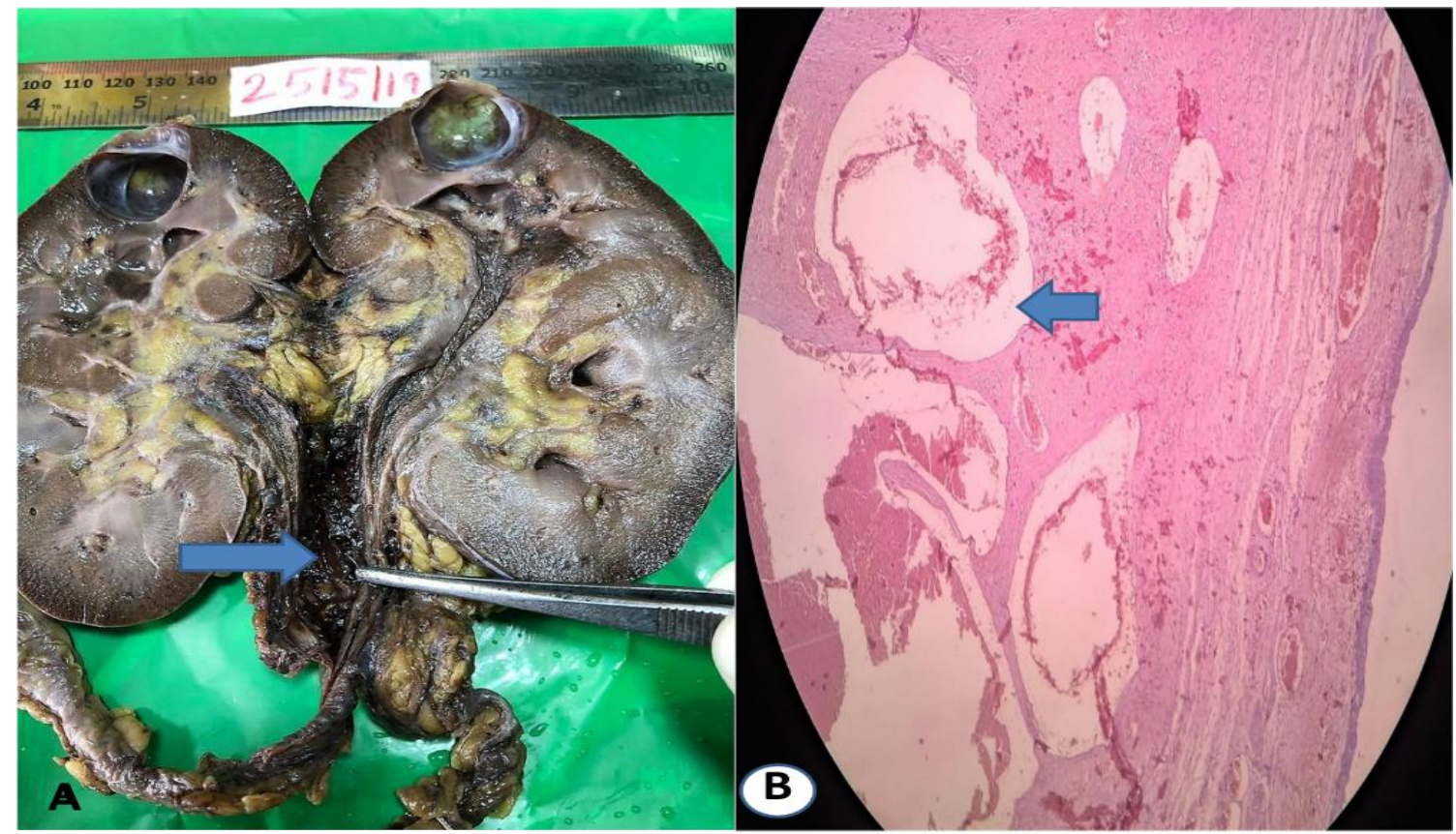

Fig. 4: Cavernous haemangioma. A- Gross specimen showing a cystic and haemorrhagic lesion in the wall of the proximal ureter (arrow). Bcavernous vascular spaces lined by endothelial cells (arrow).

Clear cell RCC was the most common (75\%) malignant tumour in our study. They are globular tumours protruding from the renal cortex, typically golden yellow, due to their cells' high lipid content (8). Fig. 5A and $5 \mathrm{~B}$ show gross and microscopic features of clear cell RCC, respectively. They are positive for PAX 8, EMA, RCC ma, CA IX, Vimentin, and CD 10 and negative for CK7 (14). Papillary RCC accounted for $13 \%$ of cases in our study. Grossly, they were predominantly cystic and haemorrhagic. Type 1 tumour was grade 2 , pT2a, and type 2 tumours were grade 2 and pT3a. The WHO/ISUP grading has supplanted the Fuhrman system and is validated for clear cell and papillary RCC. Grading is based on nucleolar prominence and must be assessed in a single highpower field having maximum pleomorphism. Both sarcomatoid and rhabdoid differentiation are regarded as grade 4 and is associated with poor prognosis (8). In the present study, grade 1 tumours were most frequent (47\%), followed by grade $2(33 \%)$ and grade $3(20 \%)$ (Fig. 5B, 5C, 5D, respectively). 


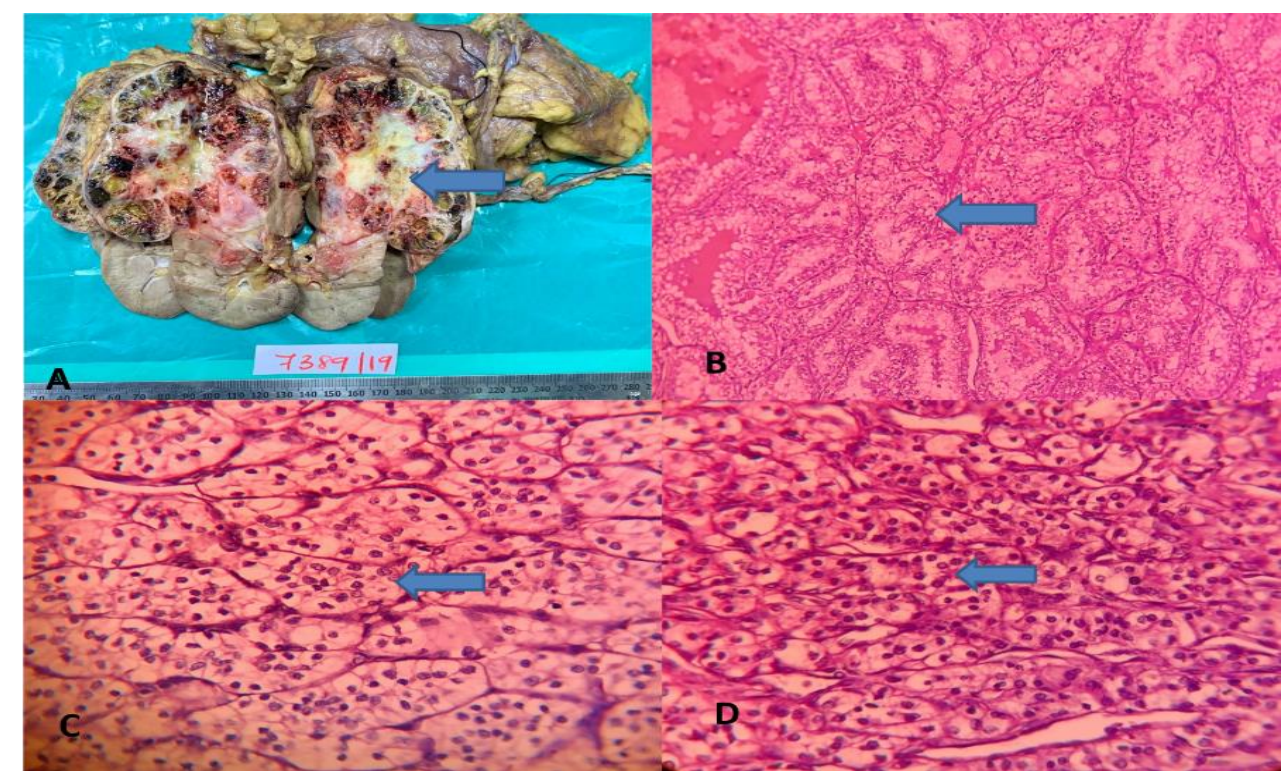

Fig. 5: Clear cell RCC. A- Gross specimen showing a golden- yellow tumour protruding from the upper pole (arrow). B-Microscopy showing tumour cells with round nucleus and inconspicuous nucleoli (arrow), Grade 1, H\&E, 40x. C-nucleoli conspicuous but not prominent (arrow), Grade 2, H\&E, 40x. D-nucleoli conspicuous and eosinophilic (arrow), Grade 3, H\&E, 40x.

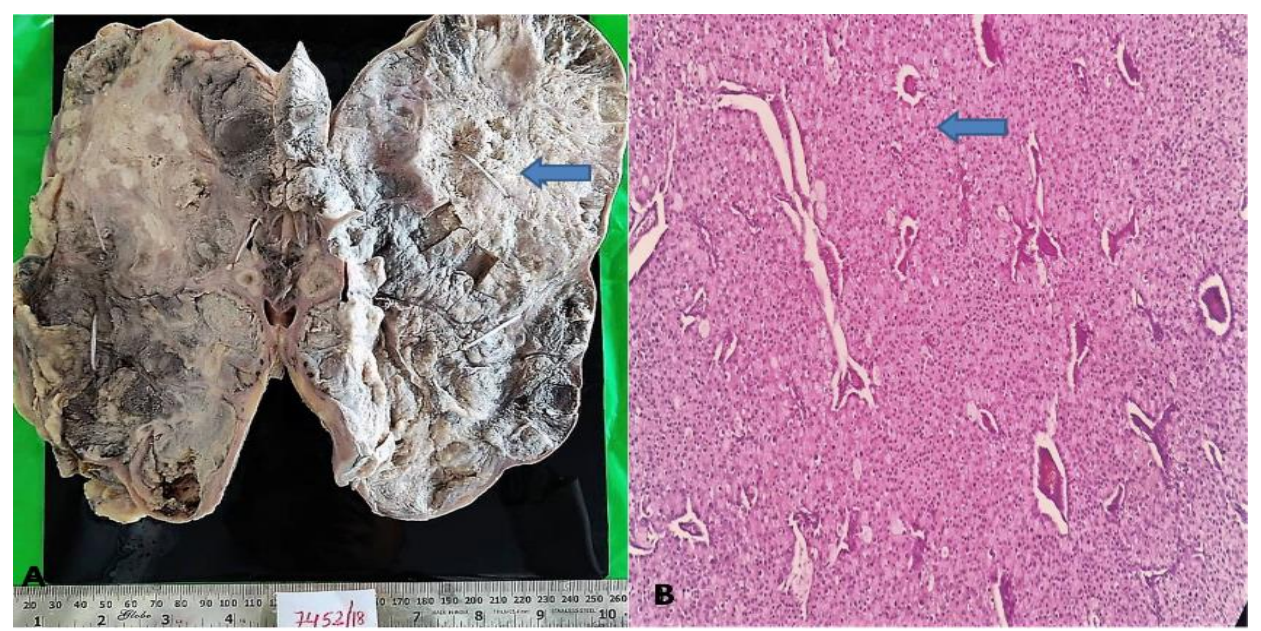

Fig. 6: Chromophobe RCC. A-Gross specimen showing grey- tan, fleshy tumour involving the entire kidney (arrow). B-Microscopy showing cells with prominent cell membranes, wrinkled nuclei with perinuclear haloes (arrow), H\&E, 10x.

Chromophobe RCC's are often large, wellcircumscribed, and unencapsulated (8). Fig. 6A and 6B shows gross and microscopic findings of Chromophobe RCC with sarcomatoid differentiation $(<20 \%)$ (Fig. 7C). This was in a 57year old female with
pT3apN1tumour with necrosis $>60 \%$, renal sinus lympho-vascular invasion (Fig. 7D), and metastatic tumour deposits in four paracaval lymph nodes. Immunohistochemistry findings are shown in Fig. $8 \mathrm{E}, 8 \mathrm{~F}$ and $8 \mathrm{G}$, and Table 3 .
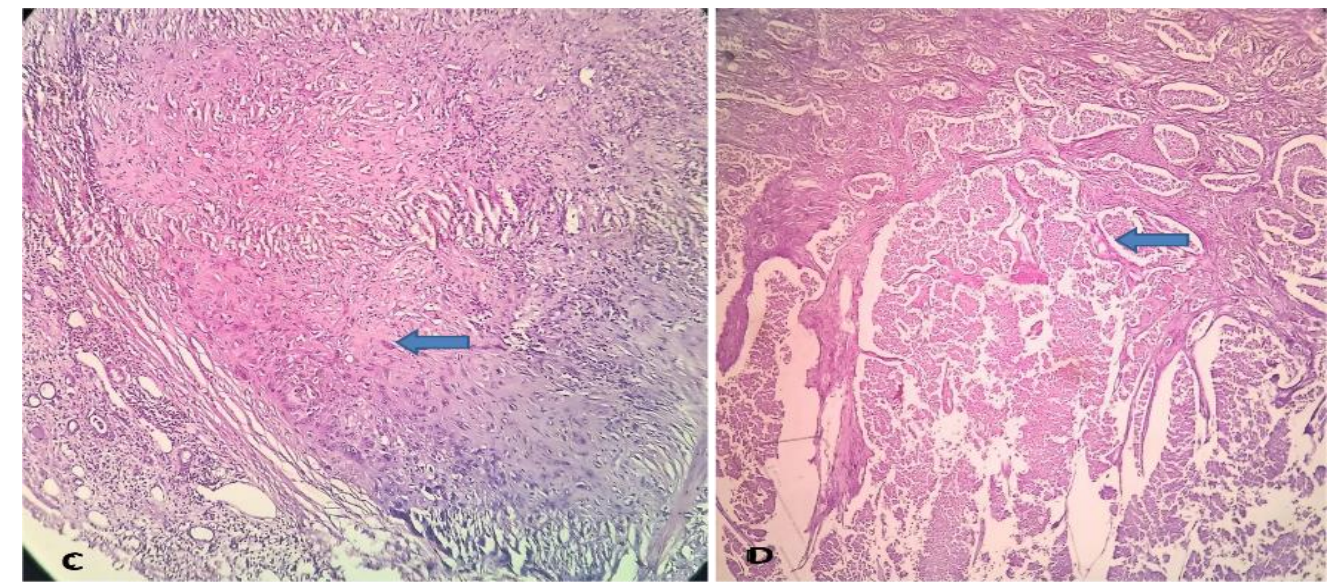

Fig. 7: Chromophobe RCC: C- Sarcomatoid differentiation (arrow), H\&E, 10x. D- Renal sinus vessel involvement, pT3a, (arrow), H\&E, 10x 


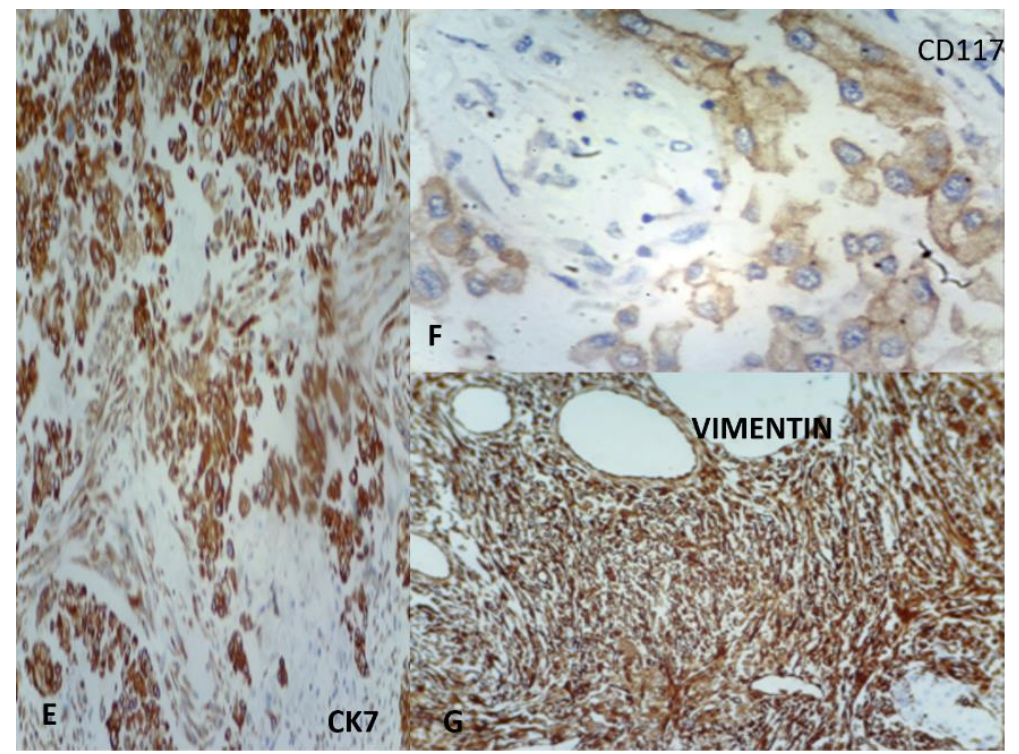

Fig. 8: Chromophobe RCCImmunohistochemistry stains E- CK 7 showing diffuse cytoplasmic positivity, F- CD 117 positive, G-vimentin positive in spindle cells.

The presence of tumour necrosis (macroscopic and microscopic) is an important prognostic factor, independent of the tumour stage and has been validated for clear cell and chromophobe RCC. Tumour necrosis accounting for $>10 \%$ of the total tumour volume, is associated with a less favourable outcome (17). In our study, the majority of them (8cases) did not show tumour necrosis. One case $(8 \%)$ showed $<10 \%$ necrosis and 4 cases $(30 \%)$ showed $>10 \%$ necrosis.

Documentation of macroscopic and microscopic extent of the tumour beyond the kidney into perinephric fat (pT3a) and Gerota's fascia (pT4) provides important staging information (18). The renal sinus is an important pathway of the spread of RCC, and its involvement (pT3a) predicts a more aggressive outcome; hence generous sampling is necessary (19). Renal sinus involvement is defined as the tumour in contact with renal sinus fat, or loose connective tissue beyond renal parenchyma or involvement of endothelial lined spaces with or without mural smooth muscle, including lymphatics. It must also be noted if the tumour is confined to the renal vein (pT3a) or extending into the inferior venacava $(\mathrm{pT} 3 \mathrm{~b} / \mathrm{c})$. It is important to note if there is a contiguous spread of the tumour to the adrenal gland (pT4) or non-contiguous involvement (pM1) (18).

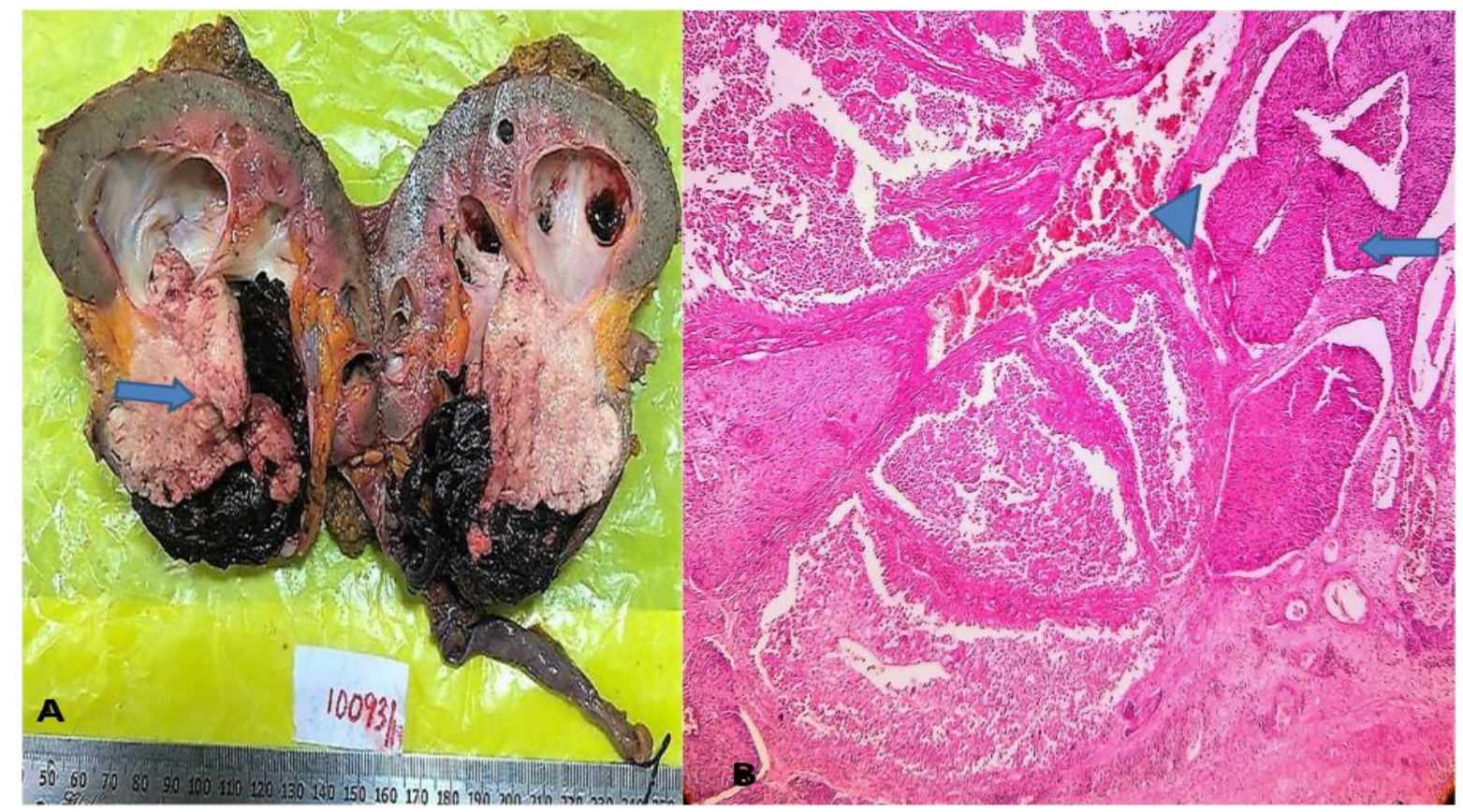

Fig. 9: High-Grade Invasive urothelial carcinoma of the pelvis A- Gross specimen showing grey white friable tumour in the renal pelvis(arrow)causing hydronephrosis. B- Microscopy showing stratified layer of tumour cells in nests (arrow) with lymphovascular invasion (arrowhead), H\&E, 10x. 


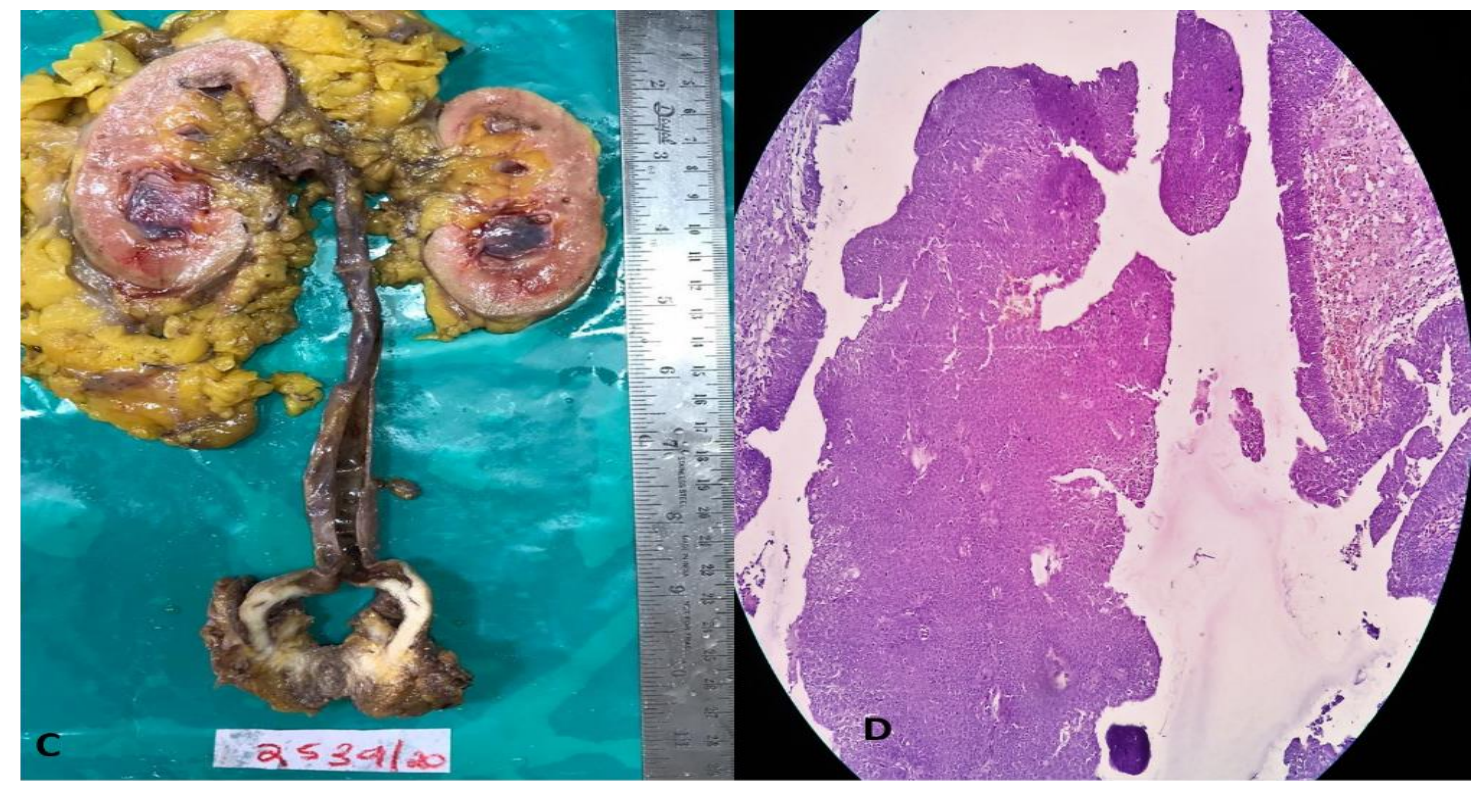

Fig. 10: High Grade Invasive urothelial carcinoma of the ureter C- Radical nephrectomy with bladder cuff resection for the tumour in the distal ureter (arrow), D- Microscopy showing HGUC (arrow), H\&E, 10X.

For assessing the surgical resected margins of malignant tumours in partial nephrectomy specimens, the renal parenchymal margin, perinephric fat margin/ renal capsular margin should be inked and examined. In radical nephrectomy specimens, ureteric, renal vein, renal artery, Gerota's fascia, and renal sinus margins should be examined (20). In the present study, one case of chromophobe RCC showed renal sinus vessel invasion. The rest of the RCCs had negative margins. Urothelial carcinomas of renal pelvis tend to be more often high grade. Significant outcome differences have been found between low and high-grade invasive tumours, and tumour grade should continue to be reported for invasive carcinoma. Infiltrative cords and single cells have the worst outcome (8). Depth of invasion, lymphovascular invasion, and pathological stage are the most important prognostic indicators for UTUC. Resection margins to be given include radial hilar soft tissue, bladder cuff, ureteral, renal parenchymal, and Gerota's fascia. Regional lymph node involvement with an extracapsular extension may be used as an indication for adjuvant therapy (21). Fig. 9A and $9 \mathrm{~B}$ shows the gross and microscopic features of the HGUC of the renal pelvis. Fig. 10C and 10D show gross and microscopic features of HGUC of the distal ureter. Regarding margin status, low-grade urothelial carcinoma of mid ureter showed adventitial tumour deposits at the ureteric margin. The rest of the two cases had a negative margin.

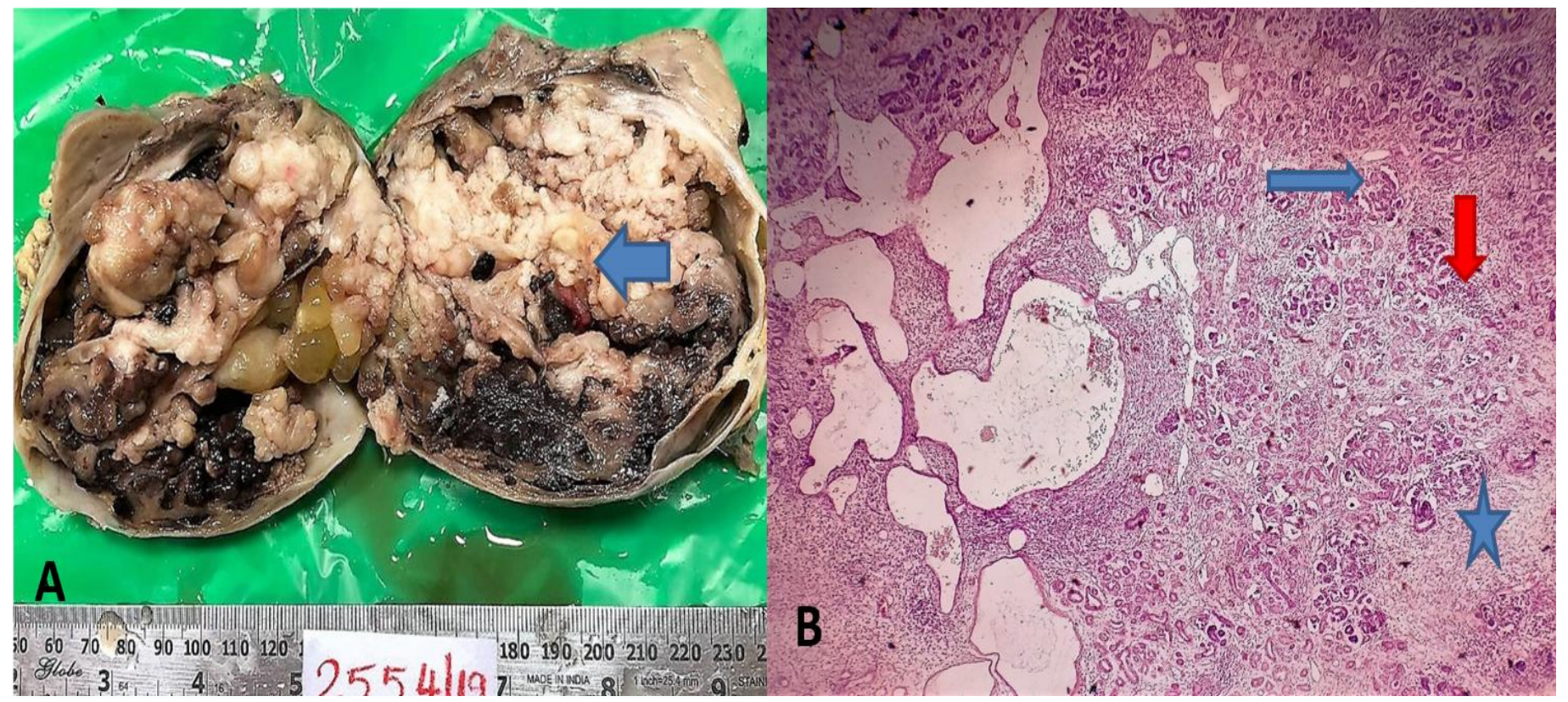

Fig. 11: Wilms' tumour A- Gross specimen showing nodular, grey, tan tumour involving the entire kidney(arrow). B- Microscopy showing epithelial (blue arrow), blastemal (red arrow), and mesenchymal elements (asterix), H\&E, 10x. 


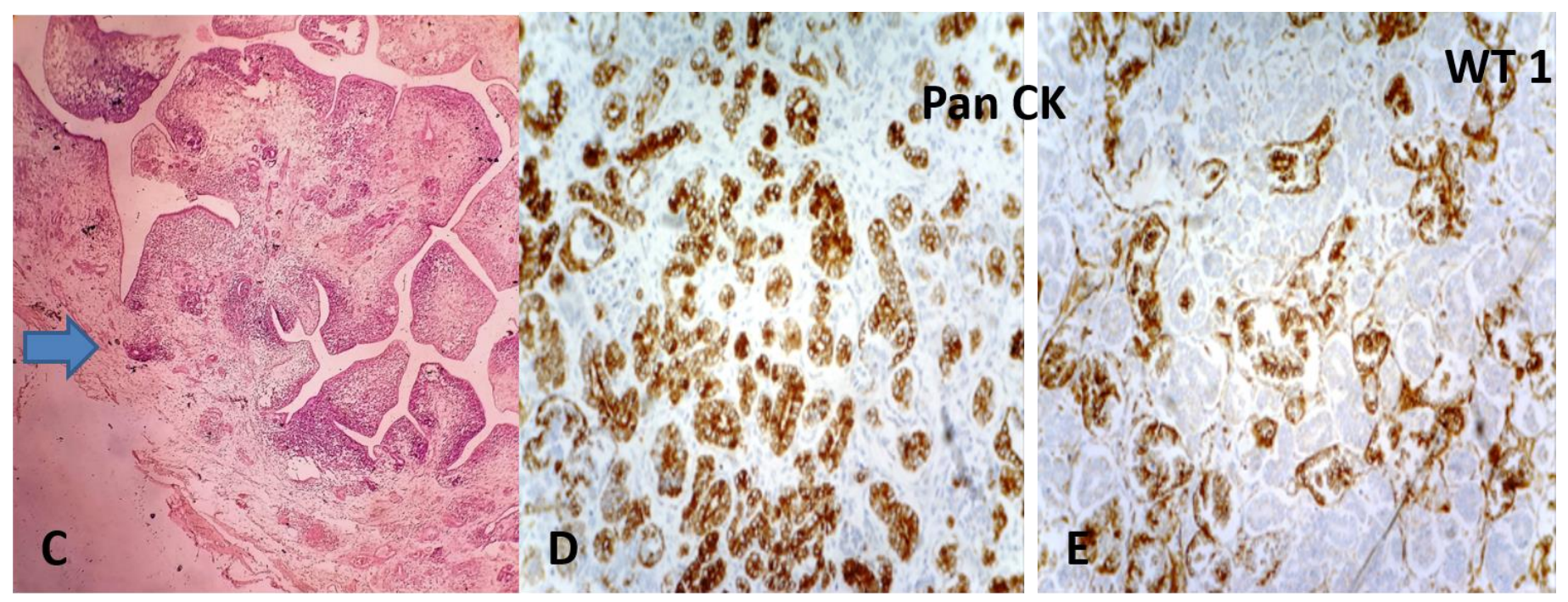

Fig. 12: Wilms' tumor C- Tumor invading gerotas fascia, stage 3 (arrow), H\&E, 10x. D- Pan CK positive in epithelial elements, E-WT 1 nuclear positivity in epithelial elements.

Nephroblastoma is a malignant embryonal neoplasm derived from nephrogenic blastemal cells. Fig. 11A and 11B shows the gross and microscopic findings of triphasic Wilms' tumour. The presence of anaplasia (focal or diffuse) is an unfavourable prognostic indicator. The children's oncology group staging is recommended to stage a Wilms' tumour. It is important to note that minimal renal sinus fat invasion and intrarenal vessel invasion do not upstage the patient to stage II. Extrarenal vascular invasion or renal sinus vessel invasion or extensive renal sinus fat invasion does upstage to stage II. The presence of nephrogenic rests (intralobular/perilobar) has to be documented as it is associated with genetic syndromes and increases the risk of developing contralateral Wilms tumour in children less than 1year (8). We had an 8month old baby with triphasic Wilms' tumour, with focal anaplasia and renal sinus fat and vessel invasion and Gerota's fascia invasion(Fig. 12C). There were no nephrogenic rests. Intraoperative tumour rupture and spill had occurred, and hence stage III was assigned. Immunohistochemistry findings are shown in Table 3 and Fig. 12D and 12E.

\section{Strengths and limitations of this study}

We have projected the various non-neoplastic and neoplastic lesions seen in nephrectomy specimens, emphasizing the pathological assessment of prognostic indicators in malignant lesions. However, we could not follow up with the patient post the pathological diagnosis as the majority opted to go to the regional cancer centre in their hometown.

\section{Recommendations}

The present study is only a retrospective case recordbased study. Hence, we would like to plan a prospective study to assess the specimens on the grossing table, and prognostic microscopic features.

\section{CONCLUSION}

Pathological evaluation is a pivotal step in diagnosing non-neoplastic and neoplastic lesions of the kidney and assessing prognostic indicators and therapeutic decisions. Extensive and meticulous sampling is critical. IHC is an adjuvant tool for the exact diagnosis of eosinophilic tumours.

\section{CONFLICT OF INTEREST}

Authors declare no conflict of interest.

\section{REFERENCES}

1. Amin, A. N., Pai, P., Upadhyaya, K. A Histopathological Spectrum of Nephrectomy Specimens in a tertiary hospital in southern India. Int J Biol Med Res 2015; 6(3): 5173-5178.

2. Shaila, N.B.S., Arasi, T. Spectrum of Lesions in Nephrectomy Specimens in Tertiary Care Hospital. Journal of Evolution of Medical and Dental Sciences 2015; 4(73): 12714-12726.

3. Abraham, G. P., Cherian, T., Mahadevan, P., Avinash, T. S., George, D., Manuel, E. Detailed study of survival of patients with renal cell carcinoma in India. Indian J Cancer 2016; 53: 572-574.

4. Mehra, M., Pramod., Gupta, N., Sharma, L. Histopathological Patterns of Renal Tumors Seen in Nephrectomy Specimens: A Three-Year Experience at a Tertiary Care Hospital in Western Part of Rajasthan Int J Med Res Prof. 2016; 2(2): 221-224.

5. Mckiernan, J., Yossepowitch, O., Kattan, M. W., Simmons, R., Motzer, R. J., Reuter, V. E., Russo, P. Partial nephrectomy for renal cortical tumors:Pathological findings and impact on outcome Adult Urology. 2002; 60(6): 1003-1009.

6. Tai, Y. S., Chiang, I. N., Huang, C. Y., Tai, H. C., Pu, Y. S. Effectiveness of different diagnostic tools for upper urinary tract urothelial carcinoma. Urological science 2015; 26(1): 57 60.

7. Robila, V., Kraft, A. O., Smith, S. C. New Entities, New Technologies, New Findings: A Review of the Cytologic Features of Recently Established Subtypes of Renal Cell Carcinoma. Cancer Cytopathol. 2019; 127(2): 79-97.

8. Humphrey, P. A., Moch, H., Reuter, V. E., Ulbright, T. M. eds. World Health Organization (WHO) Classification of 
Tumors. Pathology and Genetics of the Urinary System and Male Genital Organs. Geneva, Switzerland: WHO Press; 2016.

9. Narang, V., Garg, B., Walia, A., Sood, N., Malhotra, V. Histomorphological Spectrum of Nephrectomy Specimens- A Tertiary Care Centre Experience. Nat J Lab Med. 2016; 5(2): 51-54.

10. Leslie, S., Goh, A. C., Gill, I. S. Partial nephrectomycontemporary indications, techniques, and outcomes. Nat Rev Urol. 2013; 10(5): 275-283.

11. Latif, F., Mubarak, M., Kazi, J. I. Histopathological characteristics of adult renal tumors:a preliminary report. J Pak Med Assoc 2011; 61(3): 224-228.

12. Barkan, G. A., Wojcik, E. M., Nayar, R., Savic-Prince, S., Quek, M. L., Kurtycz, D. F. et al., The Paris system for reporting urinary cytology: the quest to develop a standardized terminology. Adv Anat Pathol. 2016; 23(4): 193-201.

13. Chandanwale, S. S., Naragude, P., Singh, M., Raj, A., Bamanikar, S., Buch, A. C. et al., Prevalence of pathological lesions in 161 nephrectomies: An experience from a teaching hospital in urban industrial area of Maharashtra. Indian J Med Spec 2020; 11: 21-27.

14. Ibrahim, S. S., Dhanabalan, R. T., Shanmuganathan, S. S., Balan, L. K., Thandavarayan, P., Ramalingam, S., et al. A Histopathological Review of Nephrectomy Specimens Received in a Referral Center in South Tamil Nadu. J Med Res Prac. 2016; 5(6): 13-19.

15. Lim, C. S., Schieda, N., Silverman, S. G. Update on indications for Percutaneous Renal Mass Biopsy in the Era of Advanced CT and MRI. American Journal of Roentgenology. 2019; 212(6): 1187-1196.

16. Iczkowski, K. A., Czaja, R. C. Eosinophilic Kidney Tumors. Old and New. Arch Pathol Lab Med. 2019; 143: 1455-1463.

17. Cheville, J. C., Lohse, C. M., Zincke, H., Weaver, A. L., Blute, M. L. Comparison of outcome and prognostic features among histological subtypes of renal cell carcinoma. Am J SurgPathol. 2003; 27: 612-624.

18. Amin, M. B., Edge, S. B., Greene, F. L., Byrd, D. R., Brookland, R. K., Washington, M. K. et al., eds. AJCC Cancer Staging Manual. $8^{\text {th }}$ ed. New York, NY: Springer; 2017.

19. Bonsib, S. M. T2 clear cell renal cell carcinoma is a rare entity: a study of 120 clear cell renal cell carcinomas. J Urol. 2005; 174: 1199-1202.

20. Trpkov, K., Grignon, D. J., Bonsib, S. N., Amin, M. B., Billis, A., Lopez-Beltran, A. et al., Handling and staging of renal cell carcinoma: the international society of urological pathology (ISUP) consensus conference recommendation. Am J Surg Pathol. 2013; 37: 1505-1517.

21. Olgac, S., Majumdar, M., Dalbagni, G., Reuter, V. E. Urothelial carcinoma of the renal pelvis: a clinicopathological study of 130 cases. Am J Surg Pathol. 2004; 28: 1545-1552. 